\title{
La industria cerámica turca
}

\author{
F. CETINOV \\ Comité de Dirección Turkish Ceramic Society Estambul Turquía
}

\begin{abstract}
La industria cerámica turca ha experimentado un gran crecimiento, tras el período de reindustrialización que se inicia en todo el país a principio de los años 50 . El peso de la empresa pública fue relevante en el surgimiento de la mayor parte de los sectores cerámicos. En los últimos años, los procesos de privatización y el cierre de algunas grandes factorías han hecho que predomine el sector privado. Se ofrecen las cifras más significativas de producción, empleo y comercio exterior de los sectores de pavimentos y revestimientos, sanitarios, vajilla, electrocerámica y refractarios. El crecimiento y las expectativas es muy diferenciado según los diferentes sectores. Los pavimentos y revestimientos se muestran especialmente dinámicos ocupando en la actualidad, con 230 millones de metros cuadrados, la $3^{\circ}$ posición en Europa , tras Italia y España. En el sector de sanitarios, Turquía con más de 10 millones de piezas en 1999 lidera la producción europea. En cuanto a los refractarios, el exceso de capacidad de producción , $60 \%$, plantea graves problemas a pesar de la importancia de algunas materias primas básicas. El sector de vajillas afronta una situación crítica. Los productos electrocerámicos muestran un desarrollo sostenido de en la mayor parte de los productos convencionales. Se describen asimismo las principales datos sobre la importante red asociativa, de formación e investigación existente en el país.
\end{abstract}

Palabras clave: pavimentos, revestimientos, vajilla, sanitario, refractarios, electrocerámica, historia cerámica

\section{Ceramic Industry in Turkey}

The Turkish Ceramic Industry has developed a rapid growth, after industrialisation from early fifties. The influence of the public companies was at the beginning critical, but the privatisation process launched during the last years promotes changes to private companies decisions. Most relevant figures for production, foreign trade and employment for ceramic sectors like tiles, roof, refractories, tableware, electroporcelain and red building brick are showed. Trends and prospective for different sectors are very differents. Tile and roofing products show specially relevant figures. Today, Turkey with 250 million square meters tiles produced in 2000, is located in the third position among European countries. Sanitary ware production is also relevant and with 10 million pieces/year, Turkey leading European production. For refractories area, the excess in production capacity, nearly $60 \%$ reveals strong problems, in spite of some good basic raw materials. Tableware shows also critical prospective due to the East Asian low production costs. Electroceramics production shows a sustained development for conventional materials. Data for R\&D institutions, professional and teaching ceramic network was also described.

Key words:tile, roof, tableware, sanitary ware, refractories, electroceramic, history.

\section{INTRODUCCIÓN}

Ante todo, en nombre de la Turkish Ceramic Society, quiero agradecer a la Sociedad Española de Cerámica su agradable invitación y su cálida acogida

Como ustedes conocen o podrán apreciar a lo largo de esta presentación, la industria Cerámica turca se ha desarrollado básicamente durante los últimos quince años, proceso que se continúa de forma ininterrumpida. La República turca está situada en Oriente Medio. Fue fundada en 1923 en territorios de Anatolia y del sudeste europeo. Actúa como puente entre Asia y Europa. Antes de suministrar algunos datos sobre la situación actual de su industria cerámica, querría dedicar unas palabras sobre la historia de nuestra tierra, de la que me siento muy orgulloso. De acuerdo con los restos cerámicos mas antiguos que se han encontrado durante las excavaciones en Anatolia, la historia cerámica en el territorio turco comienza 7000 antes de Cristo. Anatolia ha sido la sede de muchas civilizaciones como los, Urartes, Hititas, Kariyianos, Romanos, Griegos, por ese motivo, Anatolia ha sido llamada la cuna de la civilización. Tras la llegada de los turcos a Anatolia, contribuyeron al mantenimiento y desarrollo de esa rica herencia cultural. Durante el período de los
Seljúcidas, en el siglo $\mathrm{XI}$, ya se utilizaron revestimientos cerámicos en edificios monumentales como mezquitas, palacios, baños y fuente. Pero, el crecimiento mas importante de la producción cerámicas tiene lugar durante el período Otomano, especialmente a lo largo del siglo XVI. Los azulejos de Iznik pertenecen a este período con sus diseños, colores y técnicas, produciéndose aquí los primeros azulejos decorados bajo esmalte. El color turquesa fue ampliamente utilizado durante esta época y de ahí el origen de la denominación de dicho esmalte.

Cuando en 1923, se funda la República Turca, se puede decir que no puede hablarse realmente de la existencia de la cerámica turca, a excepción de algunas alfarerías. Todos los productos cerámicos, pavimentos, revestimientos y tejas eran importados de Italia, Francia. En la actualidad, puede decirse, por el contrario, que todas las ramas de la producción cerámica tienen presencia en Turquía: Ladrillos de construcción, refractarios, revestimientos cerámicos de todo tipo, sanitarios, electroporcelana, vajillas y cerámica artística. De entre todos ellos, destaca la producción de pavimentos y revestimientos cerámicos y sanitarios. 


\section{2.-LA INDUSTRIA TURCA DE PAVIMENTOS Y REVESTIMIENTOS CERÁMICOS.}

Los pavimentos y revestimientos cerámicos se importaron hasta comienzos de los años 50. En 1950 el gobierno y algunas empresas privadas, comienzan a instalar algunas fabricas cerámicas. Las empresas estatales producirían, pavimentos y revestimientos, sanitarios, electrocerámica, y vajillas. La producción comenzó en 1967, tras haber superado numerosas dificultades

La primera compañía privada en producir revestimientos fue la Canakkale Seramik Industry, que inició la producción de pavimetos en 1959 y la de electrocerámica en 1960; A lo largo de los años Canakkale Seramik ha ido creciendo, hasta convertirse en el mayor grupo productor de Turquía. En la actualidad su nombre es Kale Group y produce 61 millones de metros cuadrados en diferentes plantas situadas en la misma ciudad, lo que la convierte en uno de los mayores grupos mundiales.

\subsection{Capacidad de Producción y Empleo}

Las 20 compañías productoras, tienen una capacidad instalada de 200 millones de metros cuadrados/año y emplean a cerca de 10.000 personas, cifra en que no se incluyen a los trabajadores de las explotaciones mineras. De incluirse la cifra total alcanzaría los 12.000 empleados, 700 de ellos tienen una formación técnica a nivel universitario. De acuerdo con las estadísticas correspondientes a 1999, Turquía es el $5^{\circ}$ productor mundial tras China, Italia, España y Brasil, siendo por tanto el $3^{\circ}$ de Europa.

TABla 1. PRincipales Países PROductores en 1999 (x1000 m²)

\begin{tabular}{|l|c|}
\hline China & 1.600 .000 \\
\hline Italia & 606.000 \\
\hline España & 602.000 \\
\hline Brasil & 428.000 \\
\hline Turquía & 150.000 \\
\hline México & 130.000 \\
\hline Alemania & 64.000 \\
\hline Portugal & 60.000 \\
\hline Japón & 60.000 \\
\hline Francia & 50.000 \\
\hline
\end{tabular}

La producción turca de revestimientos se ha ido incrementando conforme al crecimiento del consumo mundial. Entre 1991 y 1999, el crecimiento anual fue cercano al 15\%. Los dos grandes terremotos de 1999 redujeron la producción, pero durante el año 2000 se han superado las cifras de 1999.

Tabla 2 Producción de Pavimentos Cerámicos e Incremento En TurQuía( Millón DE METROS CUADRAdOS/AÑO,\%)

\begin{tabular}{|l|l|l|l|l|l|}
\hline Año & Producción & Incremento & Año & Producción & Incremento \\
\hline 1990 & 52 & - & & & \\
\hline 1991 & 60 & 15 & 1996 & 120 & 13 \\
\hline 1992 & 71 & 18 & 1997 & 148 & 22 \\
\hline 1993 & 84 & 18 & 1998 & 154 & 4 \\
\hline 1994 & 93 & 11 & 1999 & 150 & -3 \\
\hline 1995 & 107 & 15 & $2000 \mathrm{e}$ & 160 & 6 \\
\hline
\end{tabular}

\subsection{Exportaciones}

Se indican los valores de la exportación y su incremento anual desde 1990 a 1999. En 1990 se exportaron 7.5 millones de metros cuadrados; en 1999 la cifra se elevó hasta 50 millones de metros cuadrados y se espera que en el 2000, haya alcanzado el valor de 56 millones de metros cuadrados. El incremento medio anual ha sido de casi el 23\%. El 50\% ha sido exportado a países europeos, el resto se envió a Estados Unidos, Canadá, países del C.I.S , llegando la exportación, en su conjunto a países de los cinco continentes.

Tabla 3. Exportación de Pavimentos Cerámicos en Turquía (TONSX1000/ AÑO)

\begin{tabular}{|l|l|l|l|l|l|}
\hline Año & Exportación & Incremento & Año & Exportación & Incremento \\
\hline 1990 & 7.5 & - & & & \\
\hline 1991 & 13 & 7.3 & 1996 & 36 & 33 \\
\hline 1992 & 14 & 7.7 & 1997 & 40 & 11 \\
\hline 1993 & 17 & 21 & 1998 & 42 & 5 \\
\hline 1994 & 19 & 12 & 1999 & 50 & 19 \\
\hline 1995 & 27 & 42 & $2000 \mathrm{e}$ & 56 & 10 \\
\hline
\end{tabular}

\subsection{Tecnología}

Casi todas las nuevas factorías construidas han adoptado las mas modernas tecnologías. Generalmente se emplean tecnologías de monococción así como sistemas rotativos de decoración en color. Se han iniciado algunas nuevas inversiones para la producción de gres porcelánico

\section{3.- INDUSTRIA TURCA DE MATERIAL SANITARIO}

La industria cerámica de sanitario comenzó su producción, al igual que la de revestimientos, a escala industrial en 1960. Eczacibasi y Yarimca Porseleen (empresa pública), han comenzado y efectuar estudios de viabilidad la mismo tiempo. Yarimca Porseleen empezaría a producir en 1967, mientras que Eczacibasi había empezado ya en 1960. Eczacibasi es el líder turco en este sector. Ahora su nombre es Vitra del Eczacibasi Group que alcanzó el $7^{\circ}$ lugar en la producción mundial, con una capacidad de 3.8 millones piezas / año. Es el líder mundial en cuanto mayor productor en una sola planta con una capacidad de 2.5 millones piezas/ año. El Grupo Kale y Roca de España han establecido el grupo Kale-Roca en que participan al $50 \%$.

\subsection{Capacidad de Producción y Empleo}

Existen más de 40 productores en Turquía. La capacidad de producción total es de 12.5 millones de piezas / año, correspondientes a $140.000 \mathrm{t} /$ año. Ocho de las compañías (con capacidades unitarias superiores a las 500.000 piezas / año), alcanzan un total de 8.5 millones de piezas/año. En 1998, se produjeron un total de 10 millones de piezas, lo que colocó a Turquía a la cabeza de la producción europea.

Tabla 4. Producción de Cerámica Sanitaria en Europa. 1998 (x1000 PIEZAS)

$\begin{array}{lr}\text { Turquía } & 10.000 \\ \text { Italia } & 9.200 \\ \text { España } & 8.100 \\ \text { Inglaterra } & 7.000 \\ \text { Francia } & 6.500 \\ \text { Portugal } & 6.400\end{array}$

En el sector trabajan aproximadamente 5.400 personas, de las que un $15 \%$ tienen formación técnica 
Tabla 5. Producción de Cerámica Sanitaria en Turquía.

\begin{tabular}{|l|l|l|}
\hline Años & t. & Incremento \% \\
\hline 1995 & 98.500 & - \\
\hline 1996 & 106.000 & 7.6 \\
\hline 1997 & 116.700 & 10 \\
\hline 1998 & 122.200 & 4.7 \\
\hline 1999 & 116.200 & -5 \\
\hline
\end{tabular}

TABla 6. EXPortaciones Turcas de CERÁmica SANITARIA

\begin{tabular}{|l|l|l|}
\hline Años & t. & Incremento \% \\
\hline 1993 & 18.400 & - \\
\hline 1994 & 30.000 & 63 \\
\hline 1995 & 31.500 & 5 \\
\hline 1996 & 38.200 & 21 \\
\hline 1997 & 46.700 & 22 \\
\hline 1998 & 45.700 & -2 \\
\hline 1999 & 50.000 & 9 \\
\hline $2000 \mathrm{e}$ & 55.000 & 10 \\
\hline
\end{tabular}

El incremento de la producción sanitaria ha sido de casi el 7\% por año, excepto en 1999.

El valor de las exportaciones se ha ido incrementado año a año, desde las 18.400 t. exportadas en 1993, esperándose que las toneladas exportadas en el 2000 sean 55.000 .

El incremento medio anual de las exportaciones, entre 1993 y el 2000, ha sido del 18\%. El $44 \%$ ha sido exportado a Alemania, el 18\% a Inglaterra, los países de la Unión Europea han supuesto en su conjunto el $70 \%$.

Tabla 7 Principales Países Exportadores de Cerámica Sanitaria en EUROPA

\begin{tabular}{|l|l|}
\hline País & $\mathrm{x} 1000$ piezas \\
\hline Portugal & 4.200 \\
\hline España & 3.500 \\
\hline Italia & 3.400 \\
\hline Turquía & 3.300 \\
\hline Francia & 2.900 \\
\hline Alemania & 1.500 \\
\hline
\end{tabular}

\subsection{Tecnología}

Los grandes productores usan métodos de alta y media presión. Algunos de ellos emplean métodos de colaje en batería. El resto emplea métodos convencionales de colaje en moldes de yeso. Se emplean regularmente robots para el esmaltado y hornos modernos de gas natural.

\section{INDUSTRIA TURCA DE ELECTROPORCELANA}

La industria de la electroporcelana se estableció en 1960, al mismo tiempo que la de pavimentos con la Factoría Can del grupo Kale y la empresa pública Yarimca Porcelain Factory. Esta última ha producido aislantes de media y baja tensión durante cerca de 30 años y posteriormente fue cerrada de acuerdo con el programa de privatización del Gobierno.

En 1970 Kaleproselen A.S. ( perteneciente al grupo Kale) inició la producción de componentes de electroporcelana como aisladores, fusibles, bujías etc.. En la actualidad el grupo Kale se ha convertido en el único productor de aisladores de alta tensión con una capacidad de producción de 10.000 t. / año, la capacidad de producción en componentes cerámicos es de 1000 t./año. En el sector se emplean 760 trabajadores.

Existen alrededor de 30 productores en Europa, con una capacidad de producción de 125.000 t./año. Algunas de las compañías pertenecen al mismo grupo, por ejemplo Ceram
Handle dispone de 7 factorías y una capacidad de 25.000 t./año.

TABla 8. CAPACIDAD INSTALADA DE PRODUCCIÓN DE ElECTROPORCELANA EN Europa (t. / año)

\begin{tabular}{|l|l|l|l|}
\hline Rusia & 25.000 & República Checa & 6.000 \\
\hline Alemania & 13.500 & Polonia & 5.000 \\
\hline Turquía & 11.000 & Francia & 4.400 \\
\hline Inglaterra & 10.500 & Austria & 4.000 \\
\hline Bulgaria & 10.000 & Bélgica & 3.300 \\
\hline
\end{tabular}

Fuente Insulator News \& Marketing Report

La industria turca de electroporcelana produce aislantes de baja tensión ( hasta $1 \mathrm{KV}$ ), media ( hasta $36 \mathrm{KV}$ ) y alta tensión ( hasta $380 \mathrm{KV}$ )

TABla 9. Producción tURCA DE ElectroporCELANA

\begin{tabular}{|l|l|l|l|}
\hline Año & Producción & Año & Producción \\
\hline 1991 & 8.600 & 1996 & 11.300 \\
\hline 1992 & 9.300 & 1997 & 8.400 \\
\hline 1993 & 11.100 & 1998 & 10.400 \\
\hline 1994 & 7.400 & $2000 \mathrm{e}$ & 9.800 \\
\hline
\end{tabular}

La utilización de la capacidad de producción se sitúa como media en le $65 \%$, oscilando entre el 50 y $80 \%$

La producción se vende generalmente a las plantas de la Turkish Electricity Authority. Se exporta únicamente el 2 o $3 \%$. La tecnología empleada es la convencional de extrusión vía húmeda.

\section{INDUSTRIA TURCA DE REFRACTARIOS.}

La industria turca de refractarios, inició sus actividades a comienzos de los años 50, produciendo sólo materiales silicoaluminosos. A partir de 1960, se fundan algunas pequeñas compañías privadas. A fines de 1968, se instala la primera planta de refractarios básicos, siendo las primeras inversiones de carácter gubernamental. Sólo tres de las compañías son propietarias de sus propias minas de materias primas; las otras compran las materias primas a productores locales o las importan de países como China, Sudáfrica, etc.

En los comienzos de la industria turca de refractarios, las compañía existentes, se especializaron en la producción exclusiva de refractarios silicoaluminosos o básicos; posteriormente y a medida que el consumo específico de refractarios se fue reduciendo en los principales sectores consumidores, siderurgia, cemento, metalurgia no férrea, las compañías comenzaron a producir indistintamente refractarios ácidos o básicos.

TABLA 10. CAPACIDAD DE PRODUCCIÓN INSTALADA POR COMPAÑÍAS (t./año). 2000

\begin{tabular}{|l|l|l|}
\hline Compañia & Magnesita sinterizada & Ladrillos+Masas \\
\hline Súperates & - & $\mathbf{9 0 . 0 0 0}$ \\
\hline Krom. Magnezit & 40.000 & 84.000 \\
\hline Kúmas & 145.000 & 80.000 \\
\hline Sórmas & - & 53.000 \\
\hline Haznedar & - & 50.000 \\
\hline Fillyos & - & 44.000 \\
\hline Magnezit A.S. & 145.000 & - \\
\hline Otras & - & 156.000 \\
\hline Total & 330.000 & 557.000 \\
\hline
\end{tabular}

En el sector trabajan 1.780 personas, de ellos 75 son ingenieros. 
Tabla 11. Producción Anual de Refractarios en Turguía (t.)

\begin{tabular}{|l|l|l|}
\hline Año & Producción (t.) & Capacidad Utilizada \% \\
\hline 1993 & 193.000 & 35 \\
\hline 1994 & 172.000 & 31 \\
\hline 1995 & 211.000 & 38 \\
\hline 1996 & 209.000 & 37 \\
\hline 1997 & 205.000 & 37 \\
\hline 1998 & 207.000 & 37 \\
\hline 1999 & 204.000 & 34 \\
\hline
\end{tabular}

TABLA 12.IMPORTACIÓN Y EXPORTACIÓN DE REFRACTARIOS (t.)

\begin{tabular}{|l|l|l|}
\hline 1993 & 52.600 & 29.000 \\
\hline 1994 & 54.000 & 20.000 \\
\hline 1995 & 70.000 & 60.000 \\
\hline 1996 & 68.000 & 49.000 \\
\hline 1997 & 60.000 & 36.000 \\
\hline 1998 & 54.000 & 30.000 \\
\hline 1999 & 50.000 & 36.000 \\
\hline $2000 \mathrm{e}$ & - & 97.000 \\
\hline
\end{tabular}

La tecnología de conformación utilizada es la del prensado en seco. Las factorías de mayor tamaño emplean grandes prensas hidráulicas y modernos hornos túnel.

\section{INDUSTRIA TURCA DE VAJILLA}

Este sector es el más antiguo de la industria cerámica turca. La principal factoría turca, productora de vajilla, es la Yildiz Porselen, establecida en 1892 en el Imperio Otomano, está situada a las orillas de Bósforo, como suministrador del palacio imperial; en la actualidad aún sigue produciendo bajo la autoridad del Parlamento turco.

Existen tres factorías y su capacidad de producción en conjunto es de 9.500 t./año 8 (36 millones de piezas/año). Los datos generales del sector se recogen en la Tabla 13

TABla 13. Estadísticas de LA INDUSTRIA tURCA DE VAJILlas (т. / AÑo)

\begin{tabular}{|l|l|l|}
\hline Año & Producción & Exportación \\
\hline 1995 & 7.080 & 415 \\
\hline 1996 & 7.600 & 315 \\
\hline 1997 & 5.850 & 730 \\
\hline 1998 & 6.000 & 1.200 \\
\hline 1999 & 6.150 & 1.130 \\
\hline
\end{tabular}

Los datos se refieren exclusivamente a la producción de vajillas de porcelana

\section{LA INDUSTRIA TURCA DE LADRILLOS Y TEJAS}

El número de empresas productoras es de cerca de 500 . Están localizadas en la zona Este y Media de Anatolia. El principal productor es Isiklar Holding y su capacidad de producción es de 300 millones de ladrillos al año. La capacidad total de producción es de 7.5 billones de piezas al año y 1 billón de piezas de tejas.

El máximo número de trabajadores es aproximadamente de 40.000, unas pocas grandes compañías trabajan todo el año, las otras trabajan sólo 7 o 8 meses al año. Los combustibles empleados son, carbón pulverizado, fuel-oil o gas natural.

\section{CONCLUSION}

El crecimiento medio anual de la producción industrial en Turquía ha sido desde 1994, del 7\% anual.

En 1998 las crisis de Asia y Rusia afectaron negativamente al mercado de la exportación, al desplazarse las inversiones de capital hacia los países en vía de desarrollo. El terremoto del 17 de Agosto supuso un verdadero desastre para la economía turca, el gobierno ha impuesto desde entonces un mayor control en la calidad de la construcción de edificios.

La inflación, que ha sido un problema crónico en Turquía durante más de 20 años, se ha visto reducida, como consecuencia del programa de estabilización puesto en marcha por el gobierno. A lo largo del 2000 se espera que no supere el $40 \%$.

Los revestimientos cerámicos y los sanitarios se han visto beneficiados por la reestructuración y expansión que han experimentado la vivienda y los edificios comerciales. Tanto la producción, como el consumo y la exportación, han crecido y se espera que esta tendencia se mantenga a lo largo de los próximos cinco años. En dicho período se espera que la exportación de pavimentos crezca en un 18\%, las ventas domésticas el $7 \%$; la exportación de sanitario crecerá el $8 \%$ y las ventas domésticas el 5\%.

De acuerdo con las previsiones del Departamento Estatal de Planificación, en los próximos cinco años la capacidad de producción del sector de pavimentos se expandirá hasta los 280 Millones de metros cuadrados y el de sanitario llegará hasta 165.000 t/año.

Tras la crisis, las industrias siderúrgicas y cementeras, han incrementado su producción y en consecuencia, ha aumentado la producción y exportación de refractarios. No obstante las compañías de refractarios disponen de un exceso de capacidad de producción y un cierto número de ellas están a la venta.

La industria de electroporcelana mantiene sus cifras de producción, mientras que la vajillas afronta una situación crítica.

La industria turca de la cerámica es mas joven que otros sectores industriales, lo que supone que dispone de un equipamiento muy moderno, también necesita una mano de obra muy cualificada. Por ello existe un elevado número de estudiantes universitarios, 2000, distribuidos en los siguientes centros:

- 3 Facultades de Ingeniería Cerámica, 8 Departamentos de Cerámica en Facultades de Bellas Artes y 10 Departamentos de Cerámica en Escuelas Politécnicas, además se ha creado un Centro de Investigación en Cerámica en Eskisehir, auspiciado por la Asociación Turca de Fabricantes de Cerámica y el Instituto Turco de Investigación Científica y Técnica.

- Las principales asociaciones del sector son: Turkish Ceramic Society, Ceramic Tile Manufacturers Association; Ceramic Sanitary Manufacturers Association, Ceramic \& Refractory Manufacturers Association y Ceramic Raw Material Manufacturers Association.

- Todos los fabricantes producen bajo las Normas Nacionales y muchos de ellos han obtenido el Certificado de Garantía de Calidad que proporciona el Instituto Turco de Normalización; en particular las compañías exportadoras poseen los certificados de normas internacionales EN, BS, DIN, KIWA, SI, WRC e ISO 9000 .

- Eczacibasi-Vitra, que es la segunda compañía productora de sanitarios del mundo, tiene el Certificado ISO 14.000.

Ahora el objetivo de la Industria Cerámica Turca es crear una imagen renovada de la cerámica turca e incrementar el valor añadido del sector. 